\title{
Nachruf auf Prof. Dr. Albrecht Blaser, Hirschhorn
}

\author{
Peter Dadam ${ }^{1} \cdot$ Klaus Küspert $^{2} \cdot$ Hans-Jörg Schek ${ }^{3}$
}

Angenommen: 7. Februar 2022 / Online publiziert: 16. Februar 2022

(c) Gesellschaft für Informatik e.V. and Springer-Verlag GmbH Germany, part of Springer Nature 2022

Am 24.10.2021 verstarb Prof. Dr. Albrecht Blaser im 89. Lebensjahr nach erfülltem Leben friedlich im Kreise seiner Familie zuhause in seinem Wohnort Hirschhorn am Neckar. Er hinterlässt seine Ehefrau Ursula, mit der er 63 Jahre verheiratet war, zwei Kinder, vier Enkel und einen 2021 geborenen Urenkel.

Albrecht Blaser wurde im Februar 1933 in Stuttgart geboren. Nach Schulausbildung teils unter den erschwerten Bedingungen des 2. Weltkriegs und Abitur begann er ein Mathematikstudium an der damaligen Technischen Hochschule Stuttgart bis zum Diplom 1957, gefolgt von wissenschaftlichen Assistenzzeiten in Hannover an der TH und schließlich wiederum in Stuttgart. Albrecht Blasers Promotion zum Dr.-Ing. erfolgte 1960 summa cum laude mit einer Arbeit über „Nichtlineare Schwingungen mit unstetiger Erregung" (Doktorvater Prof. Dr. H. Kauderer) an der Technischen Hochschule in Hannover, Fakultät für Bauwesen. Direkt im Anschluss an jene Doktoranden- und Post-docJahre trat Albrecht Blaser 1964 in die IBM Deutschland GmbH ein - und hielt der Firma 27 Jahre lang beruflich die Treue bis zum (Un-)Ruhestand, doch dazu später.

Erste IBM-Tätigkeit für ihn war die Mitarbeit in der Mathematischen Abteilung der Anwendungsentwicklung, deren Leitung er 1967 auch übernahm - einer Informatikabteilung also, wie man heute dazu sagen würde. Eines der Kerngebiete der Abteilung in Böblingen und anschließend in Stuttgart und damit auch für Albrecht Blaser lag in der Entwicklung und dem Ausbau des Scientif-

Peter Dadam

Peter@Dadam.info

Klaus Küspert

klaus.m.kuespert@gmail.com

Hans-Jörg Schek

schek@inf.ethz.ch

1 Uni Ulm, Ulm, Deutschland

2 Uni Jena, Jena, Deutschland

3 ETH Zürich, Zürich, Schweiz

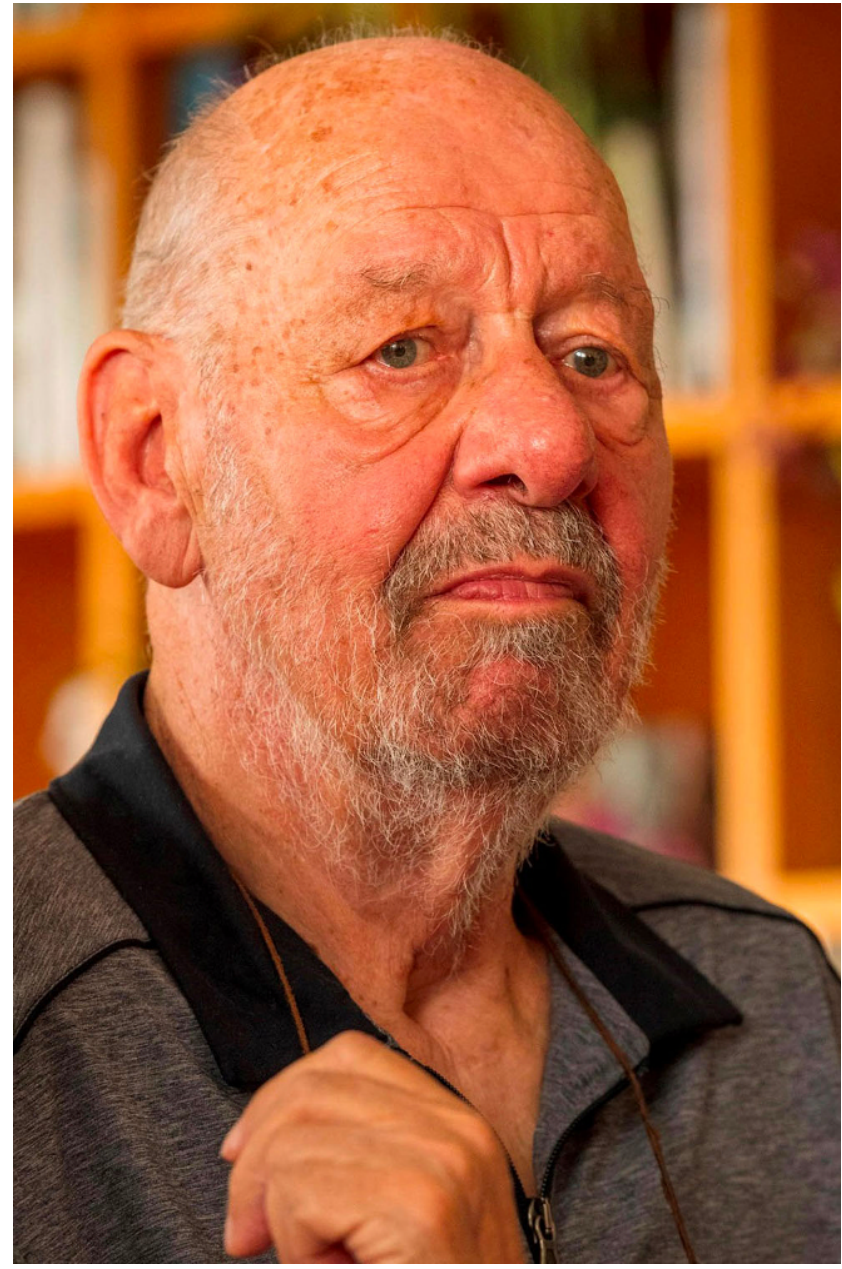

Abb. 1 Prof. Dr. Dr.-Ing. E. h. Albrecht Blaser

ic Subroutine Package (SSP), einer damals und auch anschließend über Jahre und Jahrzehnte sehr bekannten und erfolgreichen Bibliothek mathematischer und statistischer Fortran- und PL/1-Programme. Generationen von Mathematikern und Ingenieuren kamen mit SSP und dessen Verwendung in Berührung. Albrecht Blaser war in jenen Jahren ebenfalls für SSP-Nachfolgeprodukte sowie für Compilerkomponenten und weitere Teile der Anwendungsent- 
wicklung mit zuständig, dies gleichzeitig mit zunehmender Personalverantwortung im Linienmanagement der IBM. Im Anschluss in den Jahren 1972 und ' 73 verbrachte Albrecht Blaser 15 Monate als hauptamtlicher Dozent am damaligen IBM European Systems Research Institute (ESRI) in Genf (vorher schon teils als Lehrbeauftragter). Dort war er im Rahmen eines universitätsähnlich angelegten Curriculums für wesentliche Abschnitte der Mathematikausbildung bei der internen wissenschaftlichen Weiterbildung und -qualifizierung von IBM-Mitarbeitern zuständig.

1973 übernahm Albrecht Blaser schließlich die Leitung des Wissenschaftlichen Zentrums Heidelberg (WZH) der IBM und wurde dort auch später zum Direktor ernannt. Er blieb in dieser Fach- und Leitungsrolle über 17 Jahre hinweg bis 1990. Das WZH wurde in den 1970ern und '80ern unter Albrecht Blasers Leitung zur national und international anerkannten wissenschaftlichen Institution als die bedeutende Wissenschaftseinrichtung der IBM Deutschland. Das WZH war gleichzeitig IBM-Schnittstelle zum universitären Wissenschaftsbetrieb, aber auch zu großen, wissenschaftlich interessierten IBM-Kunden.

Die wissenschaftlichen Arbeitsgebiete am WZH, allesamt stark mitgeprägt durch Albrecht Blaser, können hier nur kurz angerissen werden. So ging es schon in den ersten und verstärkt in den folgenden „Blaser-Jahren" dort etwa um Endbenutzerschnittstellen zu IT- und speziell zu Datenbanksystemen, um natürlichsprachigen Datenbankzugang, um die Integration von Textverwaltung in Datenbanken (mit integrierter Abfragemöglichkeit) und dann um die Komplex-Objekt-Thematik in erweiterten relationalen Datenbanksystemen (Stichwort Non-First-Normal-Form (NF2) als Datenmodell, realisiert im Advanced Information Management (AIM) Prototype). Die Datenbanken stellten in den WZH-Jahren somit immer ganz klar einen besonderen Schwerpunkt in Albrecht Blasers Interessens- und Arbeitsgebieten dar. Einige renommierte US-IBMer sprachen teils anerkennend, teils vielleicht auch mit leichtem Anflug von Neid von den Datenbanken als „Albrecht Blaser's hobby horse“. Blasers Verantwortungsund Leitungszuständigkeiten am WZH reichten freilich deutlich weiter und deckten auch Gebiete wie Expertensystemtechnologie, Computerlinguistik, Spracherkennung, verteilte Systeme, Angewandte Mathematik mit Optimierung und Supercomputing u. dgl. ab. In Anerkennung seiner wissenschaftlich prägenden Arbeiten, speziell im Datenbanksektor, erhielt Albrecht Blaser 1980 den Ruf auf einen Datenbanklehrstuhl an die TU Braunschweig, entschied sich aber fürs Bleiben bei IBM.

Mit der politischen Wende in der DDR 1989/90 wurde Albrecht Blaser zusätzlich, und dann 1990/91 sehr bald hauptamtlich, für die Wissenschaftsbeziehungen und -zusammenarbeit der IBM Deutschland mit den Universitäten in der DDR und anschließend den Neuen Bundesländern verantwortlich. Er förderte hier wesentlich die Ausstattung von Universitätsrechenzentren durch aktuelle (IBM-)Großrechentechnik sowie den Aufbau von PC-Pools für die Lehre an den Fakultäten. Dies war alles oftmals verbunden mit wissenschaftlichen Kooperationsprojekten zwischen der jeweiligen Hochschule und IBM gleich ab 1990/91.

1991 wechselte Albrecht Blaser dann ganz auf die Hochschulseite. Mit vorgezogenem IBM-Ruhestand wurde er für jeweils circa drei Jahre in Vollzeit als Lehrstuhlvertreter erst an der Universität Jena, dann an der Universität Heidelberg und schließlich an der TU Ilmenau aktiv. Sowohl in Thüringen als auch in Baden-Württemberg wurde er entsprechend zum Honorarprofessor ernannt. Ilmenau verlieh ihm für seine Leistungen und sein wissenschaftliches und gesamtuniversitäres Engagement darüber hinaus 2003 den Dr.-Ing. E. h.

Ganz wesentliche tiefe Spuren hinterließ Albrecht Blaser in den 1980er und '90er Jahren in der GI und den dortigen Kontexten. Dies betraf u. a. seine Mitgliedschaft im Präsidium und als Präsidiumsbeauftragter sowie insbesondere Leitungsfunktionen im damaligen GI-Fachbereich 2 „Softwaretechnologie und Informationssysteme“, im zugehörigen GI-Fachausschuss 2.5 „Rechnergestützte Informationssysteme“ und im GI-Ausschuss ,Forschung und Technologie“. Albrecht Blaser war zudem ab 1983 einer der tatkräftigen Initiatoren und Väter der BTW-Tagungsreihe, damals noch als „Datenbanksysteme für Büro, Technik und Wissenschaft". Dies manifestierte sich dann auch in seiner Mitherausgeberschaft sogleich des ersten BTW-Tagungsbands in Karlsruhe 1985. Nicht zuletzt ging auch die Deutsche Informatik-Akademie (DIA) als Tochter der GI auf Albrecht Blaser und einen von ihm geleiteten GI-Präsidiumsarbeitskreis zurück. Für die DIA war er dann auch ab Mitte 1990er für mehr als ein Jahrzehnt prägend und erfolgreich als wissenschaftlicher Berater und Programmverantwortlicher tätig bis 2008. Die GI ernannte Albrecht Blaser für seine Verdienste 2002 als einen der Ersten zu ihrem Fellow. Erst mit langsamer weiterer Richtung auf den 80 . Geburtstag zog sich Albrecht Blaser sukzessive aus den GIRollen und drumherum zurück, blieb aber durch Veranstaltungsteilnahmen und stets sichtbares Fachinteresse weiter der Informatik verbunden. Sein 75. Geburtstag wurde mit einem wissenschaftlichen Festkolloquium an der Universität Jena begangen.

Mit Albrecht Blaser verlieren die GI, die wissenschaftliche Gemeinde und speziell die Datenbank-Community eine ihrer über Jahrzehnte hinweg prägenden Persönlichkeiten. Viele Weggefährten, Freunde, Mitarbeiter und Schüler Albrecht Blasers haben ihn stets als hoch integre und fördernde Persönlichkeit kennengelernt und geschätzt, als einen Mentor und besonderen Menschen. Sie werden ihn stets in besonders wertschätzender Erinnerung behalten. 